\title{
基于二元 Copula 函数的沪深股市相依结构研究
}

\section{Research on dependence structure of Shanghai and Shenzhen Stock Markets based on Binary Copula function}

\author{
孙志宾 王同 \\ Zhibin Sun Tong Wang \\ 北方工业大学 北京 100144
}

North China University of Technology Beijing 100144

\begin{abstract}
摘 要: 沪深股市已经成为我国证券市场中重要的一部分,科学刻画其相依结构具有重要的现实意义。本文选取 2000 年 1 月 4 日至 2020 年 8 月 28 日的上证指数和深证综指的日收益率,分析其描述性统计表,发现都具有下列共同特征: 尖峰、厚尾 和拒绝正态分布。可以采用半参数估计法进行拟合, 首先通过分析沪深股市的样本经验分布函数和核函数,绘制沪深股市的 二元频率直方图,沪深股市的日收益率序列的尾部基本对称,因此选用正态 Copula 函数和 t-Copula 函数,描述沪深股市之间 的相依结构, 然后借助核分布估计求取二元 Copula 函数中的未知参数,最后用秩相关系数对模型进行检验。
\end{abstract}

\begin{abstract}
The Stock market of Shanghai and Shenzhen has become an important part of the stock market in Our country. It is of great practical significance to describe its dependent structure scientifically. In this paper, the daily returns of Shanghai Stock exchange index and Shenzhen Stock exchange composite index on January 4, 2000 and August 28, 2020 are selected, and the descriptive statistical tables are analyzed. It is found that they all have the following common characteristics: peak, thick tail and rejection of normal distribution. Half a parameter estimation method may be used for fitting, first of all, through the analysis of the samples of Shanghai and shenzhen stock market empirical distribution function and kernel function, the dual frequency histogram map of these Stock Markets, these stock markets return sequence is the tail of the basic symmetry, so choose normal copulas connect function and $\mathrm{t}$ - copulas connect function, describe the dependence structure between these stock markets, and then with the help of a kernel density estimation to calculate binary copulas connect function of unknown parameters, the final test with rank correlation coefficient of the model.
\end{abstract}

关键词: 沪深股市;二元 Copula; 相依结构;秩相关系数

Keywords : Shanghai and Shenzhen Stock Markets; binary Copula; dependency structure; rank correlation coefficient

DOI : $10.36012 /$ emr.v2i6.2995

\section{1.引言}

如今, 经济全球化的进程不断推进,不同国家之间的金 融市场、同个国家的不同金融市场之间,都存在着一定的关 系, 且趋于复杂化和多元化, 身处金融市场的各方都需要研 究和了解股市间复杂的相依结构,促进金融市场稳定快速的 发展。随着我国证券市场的发展, 沪深股市已经成为投资市 场的重要部分。因此, 深入地研究沪深股市之间的相依结构, 有利于股市的投资者合理投资,也有利于监管部门的宏观调 控和防范金融风险。

1990 年, Embrechts 首先将 Copula 理论应用在金融领 域, 经过多年的探索研究, Copula 函数如今得以广泛应用, 尤 其在刻画金融市场间的相依结构、资产定价、测量金融市场
风险、选择投资组合等方面贡献了重要力量。Copula 函数能 够很好的处理非线性、非对称的数据, 因此广泛应用于两个 或多个事务之间的相关分析, 还可以应用于多变量建模。 Copula 函数总体可以分为三类: 正态分布的 Copula 函数, $\mathrm{t}-$ Copula 函数和 Archimedean Copula 函数。

本文选取 2000 年 1 月 4 日至 2020 年 8 月 28 日的上证 指数和深证综指, 通过研究分析两个时间序列, 发现两者都 表现为尖峰、厚尾、拒绝正态分布的特征。研究沪深股市之间 的相依结构, 首先通过分析沪深股市日收益率序列的样本经 验分布函数和核函数, 绘制沪深股市的二元频率直方图, 从 而选取适合的二元 Copula 函数, 利用半参数估计法估计参 数, 然后根据秩相关系数对模型进行分析, 得出结论。 


\section{2.模型构建}

\subsection{Copula 函数}

定义 $1 \mathrm{~N}$ 元 Copula 函数是指定义域为 $[0,1]^{\mathrm{N}}$ 的函数 $\mathrm{C}$ $\left(\mathrm{u}_{1}, \mathrm{u}_{2}, \cdots, \mathrm{u}_{\mathrm{N}}\right)$ 满足

i. $\mathrm{C}\left(\mathrm{u}_{1}, \mathrm{u}_{2}, \cdots, \mathrm{u}_{\mathrm{N}}\right)$ 为递增的函数,且为零基面;

ii. $\mathrm{C}\left(\mathrm{u}_{1}, \mathrm{u}_{2}, \cdots, \mathrm{u}_{\mathrm{N}}\right)$ 的边缘分布函数 $\mathrm{C}_{\mathrm{i}}\left(\mathrm{u}_{\mathrm{i}}\right)$ 满足

$\mathrm{C}_{\mathrm{i}}\left(\mathrm{u}_{\mathrm{i}}\right)=\mathrm{C}\left(1, \cdots, 1, \mathrm{u}_{\mathrm{i}}, 1, \cdots, 1\right)=\mathrm{u}_{\mathrm{i}}$

其中, $\mathrm{u}_{\mathrm{i}} \in[0,1], \mathrm{i}=1,2, \cdots, \mathrm{N}$ 。

定理 1 对于边缘分布函数 $\mathrm{F}_{1}, \cdots, \mathrm{F}_{\mathrm{n}}$ 的联合分布函数 $\mathrm{F}$, 当 $\mathrm{F}_{1}, \cdots, \mathrm{F}_{\mathrm{n}}$ 均为连续的, 一定存在且唯一存在 Copula 函数 $\mathrm{C}$,使得下式成立：

$\mathrm{F}\left(\mathrm{x}_{1}, \mathrm{x}_{2}, \cdots, \mathrm{x}_{\mathrm{N}}\right)=\mathrm{C}\left(\mathrm{F}_{1}\left(\mathrm{x}_{1}\right), \cdots, \mathrm{F}_{\mathrm{N}}\left(\mathrm{x}_{\mathrm{N}}\right)\right) 。$

由上述定义 1 和定理 1 可得, Copula 函数具有存在性, 而且可以是唯一存在的, 借助 Copula 函数, 可以通过研究边 缘分布函数, 得到其联合分布函数。

\section{2 相关性度量}

目前, 测量各个随机变量之间相关性的方法较多, 其中 广泛应用的的有以下三种方法: 线性相关系数、秩相关系数 及尾部相依系数。其中前两种相关系数是测量两个不同变量 之间的相依结构, 后一种相依系数是测量同一个变量的上、 下尾之间的相依关系。由于金融数据的非线性, 则不选用线 性相关系数, 下面重点介绍两种秩相关系数, 为 Kendall 秩相 关系数和 Spearman 秩相关系数。

定义 2 设 $\left(X_{1}, Y_{1}\right),\left(X_{2}, Y_{2}\right)$ 是相互独立并且与 $(X, Y)$ 具有 相同分布的二维随机变量, 则 $\mathrm{X}$ 与 $\mathrm{Y}$ 的 Kendall 秩相关系数 $\tau$ 为:

$\tau=\mathrm{P}\left(\left(\mathrm{X}_{1}-\mathrm{X}_{2}\right)\left(\mathrm{Y}_{1}-\mathrm{Y}_{2}\right)>0\right)-\mathrm{P}\left(\left(\mathrm{X}_{1}-\mathrm{X}_{2}\right)\left(\mathrm{Y}_{1}-\mathrm{Y}_{2}\right)<0\right)$

其中, $\mathrm{P}\left(\left(\mathrm{X}_{1}-\mathrm{X}_{2}\right)\left(\mathrm{Y}_{1}-\mathrm{Y}_{2}\right)>0\right)$ 表示它们和谐的概率, 用 $\mathrm{P}$ $\left(\left(X_{1}-X_{2}\right)\left(Y_{1}-Y_{2}\right)<0\right)$ 表示它们不和谐的概率。根据该相关系 数的定义可知, 当随机变量 $X 、 Y$ 同时严格递增或者同时严 格单调递减时, $\mathrm{X} 、 \mathrm{Y}$ 之间的 Kendall 秩相关系数保持不变。

定义 3 设 $\left(X_{1}, Y_{1}\right),\left(X_{2}, Y_{2}\right),\left(X_{3}, Y_{3}\right)$ 是相互独立并且与 $(X$, $\mathrm{Y})$ 具有相同分布的二维随机变量, 定义 $\mathrm{X}$ 与 $\mathrm{Y}$ 的 Spearman 秩相关系数 $\rho$ 为:

$\rho=3\left\{\mathrm{P}\left(\left(\mathrm{X}_{1}-\mathrm{X}_{2}\right)\left(\mathrm{Y}_{1}-\mathrm{Y}_{3}\right)>0\right)-\mathrm{P}\left(\left(\mathrm{X}_{1}-\mathrm{X}_{2}\right)\left(\mathrm{Y}_{1}-\mathrm{Y}_{3}\right)<0\right)\right\}$

该相关系数实际为 $\left(X_{1}, Y_{1}\right),\left(X_{2}, Y_{3}\right)$ 和谐的概率, 减去其 不和谐的概率所得结果的 3 倍, 与 Kendall 秩相关系数一样, 当 $\mathrm{X} 、 \mathrm{Y}$ 同时严格单调递增或者同时严格单调递减时, $\mathrm{X} 、 \mathrm{Y}$ 之间的 Spearman 秩相关系数不发生变化。

\section{3.实证分析}

\section{1 样本数据及统计特征}

选取 2000 年 1 月 4 日至 2020 年 8 月 28 日上证指数和 深证综指的日收盘价, 共 5006 组有效数据, 数据来源于网易 财经。考虑到实际计算的收益率数字水平太小, 则放大十倍, 采用一阶对数差分并乘以 100 :

$$
\mathrm{R}_{\mathrm{t}}=100 \times\left(\ln \mathrm{P}_{\mathrm{t}}-\ln \mathrm{P}_{\mathrm{t}-1}\right)
$$

从而得到对数收益率序列, 上式中的 $\mathrm{P}_{\mathrm{t}}$ 代表第 $\mathrm{t}$ 天的上 证指数或者深证综指的日收盘价。通过分析两个序列的数 据, 得到上证指数和深证综指的日收益率时序图如图 1 所 示, 描述性统计结果如表 1 所示。

\section{图 1 日收益率时序图 (2000.01.04 至 2020.08.28)}
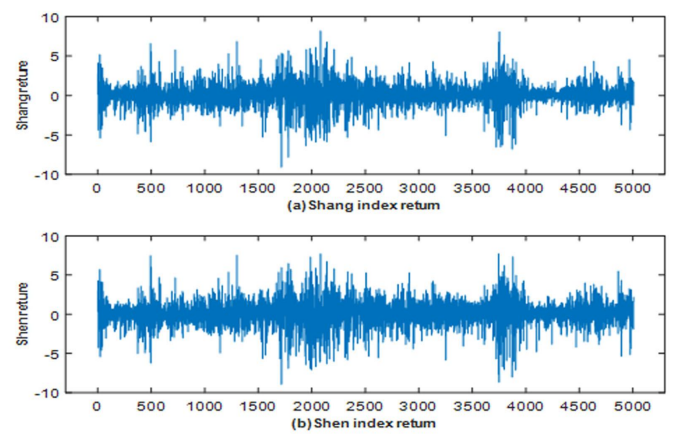

表 1 描述性统计表

\begin{tabular}{|c|c|c|c|c|c|c|}
\hline 指标名称 & $\begin{array}{c}\text { 样本数 } \\
\text { 量 }\end{array}$ & 均值 & 标准差 & 偏态 & 峰态 & $\begin{array}{c}\text { J-B 统计量 } \mathrm{p} \\
\text { 值 }\end{array}$ \\
\hline 上证指数 & 6500 & 0.0913 & 1.4180 & -0.1543 & 6.6996 & 0.001 \\
\hline 深证综指 & 5006 & 0.1182 & 1.6199 & -0.3342 & 5.8529 & 010.0 \\
\hline
\end{tabular}

通过图 1 和表 1 可得,两个序列也具有如上这些特征。沪深 股市的日收益率的均值都大于零, 但接近于 0 , 并且深证综 指的均值略大于上证指数的均值; 两个序列的标准差都大于 0 , 表现出较强的波动性; 偏态都小于 0 且峰态大于 5 , 说明 序列尖峰厚尾; 根据 J-B 统计量的检验结果, 拒绝服从正态 分布的原假设。

综上所述, 上证指数和深证综指的日收益率序列, 都存 在尖峰、厚尾、拒绝正态分布等特征,为了更好的研究沪深股 市的相依结构,选用半参数估计法进行分析。

\section{2 参数估计}

在现实研究的过程中, 边缘分布函数中可能会包含未知 的参数, 所选择的 Copula 函数中也可能会含有未知参数, 则 需要估计这些参数。目前, 应用较多的参数估计的方法有以 下三种, 最为常用的是最大似然估计方法, 此外还有分步估 
计和半参数估计 (CML 估计)两种方法。CML 估计可以不求 取边缘分布函数, 用样本经验分布函数求联合密度函数, 这 样则不需估计边缘分布函数的未知参数, 只需要计算所选择 的 Copula 函数中的未知参数, 就可以得到结论。因此, 我们 采用半参数估计的方法进行参数估计。

图 2 上海、深圳股市的经验分布函数和核分布估计图
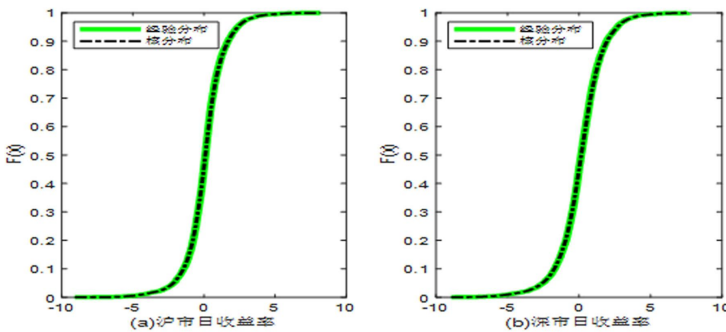

通过计算,得到如图 2 上海、深圳股市的经验分布函数 和核分布估计图。根据图 2 可知, 经验分布函数与核分布估 计的结果基本一致,则拟合的效果令人满意。

图 3 二元频数直方图

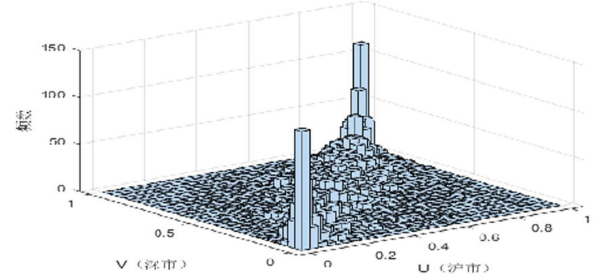

图 4 二元频率直方图

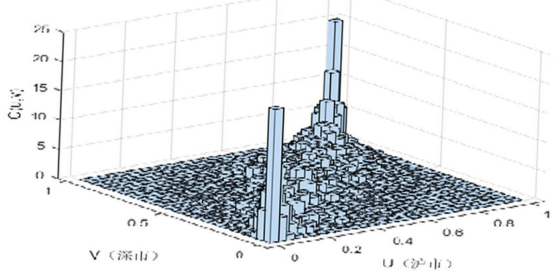

可以根据经验分布函数和核分布估计结果, 确定沪市的 边缘分布 $U=F(x)$ 和深市的边缘分布 $V=G(x)$, 然后根据 $\left(U_{i}, V\right.$ )$(i=1, \cdots, n)$ 的二元直方图, 选择合理的 Copula 函数进行估计。 得到如图 3 的二元频数直方图后, 可以继续绘制如图 4 的 二元频率直方图, 得到的二元函数可以作为沪深股市的联合 密度函数的估计。

根据二元频率直方图可知, 上证指数和深证综指的日收 益率序列之间具有基本对称的尾部, 则 $(\mathrm{U}, \mathrm{V})$ 的联合密度函 数的尾部也是对称的, 因此本文选择二元正态 Copula 函数
和二元 $\mathrm{t}-$ Copula 函数来刻画上海与深圳股市之间的相依结 构。

下面利用核分布估计,求出 Copula 函数中的未知参数 如下:

二元正态 Copula 函数: ${ }_{\mathrm{P}}=\begin{array}{ll}1.0000 & 0.9133 \\ 0.9133 & 1.0000\end{array}$

则描述上海、深圳股市之间相依结构的正态 Copula 函 数为

$\hat{C}(\hat{u}, \hat{v})=\int_{-\infty}^{\phi^{-1}(\hat{u})} \int_{-\infty}^{\phi^{-1}(\hat{v})} \frac{1}{2 \pi \sqrt{1-0.9133^{2}}} \exp \left\{-\frac{s^{2}-2 \times 0.9133 s t+t^{2}}{2 \times\left(1-0.9133^{2}\right)}\right\} d s d t$

二元 $\mathrm{t}$-Copula 函数: ${ }_{\mathrm{P}}=\left(\begin{array}{ll}1.0000 & 0.9218 \\ 0.9218 & 1.0000\end{array}\right)$

则描述上海、深圳股市之间相依结构的 $\mathrm{t}-$ Copula 函数为

$\hat{c}(\hat{u}, \hat{v})=\int_{-\infty}^{t_{+}^{-1}(\hat{u})} \int_{-\infty}^{t_{+}^{-1}(\hat{v})} \frac{1}{2 \pi \sqrt{1-0.9218^{2}}}\left[1+\frac{s^{2}-2 \times 0.9218 s t+t^{2}}{4 \times\left(1-0.9218^{2}\right)}\right]^{-(4+2) / 2} d s d t$

绘制沪深股市之间相依结构的正态 Copula 函数、t-Copula 函数的密 度函数图, 如图 5 、图 6 所示。

图 5 二元正态 Copula 密度函数图

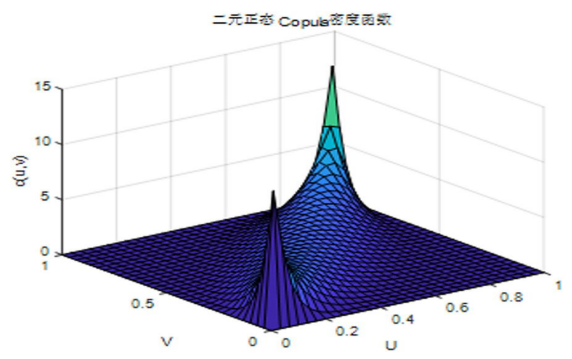

图 6 沪深股市的二元 $\mathrm{t}-$ Copula 密度函数图

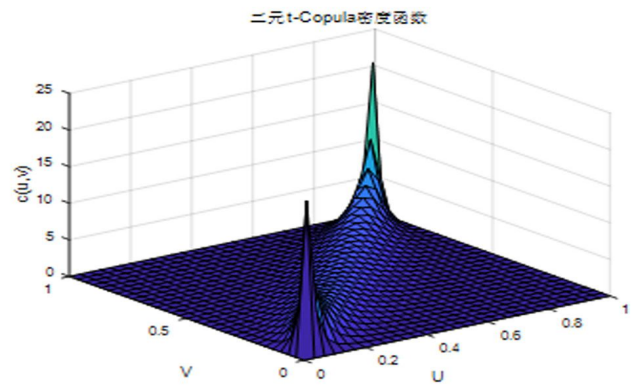

\section{3 模型评价}

表 2 Kendall 秩相关系数和 Spearman 秩相关系数

\begin{tabular}{|c|c|c|}
\hline 名称 & Kendall & Spearman \\
\hline 原始预测 & 0.7442 & 0.8986 \\
\hline 正态 Copula & 0.7330 & 0.9057 \\
\hline t-Copula & 0.7465 & 0.8996 \\
\hline
\end{tabular}


通过上述估计的参数, 可以求得正态 Copula 模型、tCopula 模型及原始预测的 Kendall 秩相关系数和 Spearman 秩相关系数, 如表 2 所示。沪深股市之间存在很强的相关 性, 通过比较原始序列预测和正态 Copula 模型、t-Copula 模 型的秩相关系数, 发现二元 $\mathrm{t}-$ Copula 函数模型的两种秩相关 系数, 都比正态 Copula 函数模型的, 更接近于原始预测的秩 相关系数, 所以得到结论: 从 Kendall 秩相关系数和 Spearman 秩相关系数的角度考虑, 二元 $\mathrm{t}-$ Copula 模型的效果更好, 能 更好地刻画沪深股市之间的相依结构。

\section{4.结束语}

通过分析近二十年的上证指数和深证综指的日收益率, 最终得到结论: 上证指数和深证综指的日收益率时间序列都 为尖峰、厚尾、拒绝正态分布; 二元 $\mathrm{t}-$ Copula 函数和二元正态 Copula 函数都可以刻画沪深股市之间的相依结构, 但从秩相 关系数的角度分析, $\mathrm{t}-$ Copula 模型比正态 Copula 模型的拟合 效果更好; 沪深股市之间存在很强的相依结构。

\section{参考文献}

[1] 何敏园,李红权.全球股市间的相依结构与极值风险溢出:基于
藤 Copula 的金融复杂性分析[J]. 管理评论,2020,32(07):102110.

[2] 刘超,王淑娇, 刘宸琦, 刘思源. 国际股票市场风险传染效应研 究——来自 2007 2018 年 15 个股票市场数据[J].复杂系统与 复杂性科学,2020,17(02):54-66.

[3] 郭森,孙志宾. 用广义帕累托分布估计极端尾部风险一以上 海、深圳股市为例[J].中小企业管理与科技(中旬刊),2018(11): 65-66.

[4] 卢俊香,武宇,杜艳丽. 基于 Copula 的沪深股市相依结构与相关 模式研究[J].四川理工学院学报(自然科学版),2016,29(02):7074.

[5] 操颙,方兆本. 基于混合 copula 函数的沪深股市与香港股市一 体化趋势分析[J].中国科学技术大学学报,2014,44(06):508515.

[6] 郭文伟,钟明.基于 Vine Copula 的中国股市风格资产相依结 构特征及组合风险测度研究[J]. 管理评论,2013,25(11):41-52.

[7] 李宝良. 基于 $\mathrm{t}$ Copula 的中国股市指数组合 VaR 估计及其 评价[J].重庆工商大学学报(自然科学版),2012,29(12):38-43+ 54.
(上接第 9 页)

\section{1 结论}

中哈农业贸易呈现波动状态但较为稳定, 中国在两国贸 易中较长时间为贸易顺差, 两国存在农产品贸易规模偏小和 农产品结构低下等问题,都需要有待改善。扩大中哈两国地 区农产品出口贸易市场,优化两国农产品贸易结构,提高两国 地区农产品出口贸易实现结构性优势互补,提高农产品贸易 规模等是中哈两国共同努力的方向和目标。

\section{2 建议}

(1)优化出口农产品的结构。中国对哈国的出口大多数 为大宗石油类产品, 对于其他动植物油的农业出口还是依然 存在很大的市场潜力, 所以中国在原来农业出口的基础上,积 极考虑去大力发展其他各种农产品加工作物的农业出口,加 强两个大国的其他农产品作物贸易合作联系, 使两个大国之 间农产品作物贸易联系达到一个更高的技术层次。
(2)强化中哈农业合作的政策保障。中哈双方相关主管 部门提出要进一步积极推动中哈海关贸易合作框架协议等 相关政策的贯彻落实, 来有效促进中哈之间农业贸易合作, 减 少中哈贸易壁垒,来大大提高中哈农业贸易合作便利。

\section{参考文献}

１１］张苏坦. “一带一路” 背景下中国与哈国农产品贸易潜力研究 [D].中国石油大学(北京),2018.

[2] 徐雅, 伍晓光. “一带一路” 倡议下中哈贸易的现状与前景 [J]. 求 索,2018(05):56-63.

[3] 刘鸣双. 中哈农业合作的发展现状及对策 [J]. 对外经贸,2020 (08):9-12.

[4] 赵晓洁.中国对哈萨克斯坦农产品出口潜力分析[D].兰州财经 大学, 2019 .

[5] 张小东, 海兰, 马岩祥. “丝绸之路经济带” 背景下中哈产能合作 的金融支持与风险防范[J]. 对外经贸实务, 2019(09):50-53. 